\title{
Reconstruction of multidimensional data in pattern analysis
}

\begin{abstract}
Proposed method, called Probabilistic Features Combination (PFC), is the method of multi-dimensional data modeling, extrapolation and interpolation using the set of highdimensional feature vectors. This method is a hybridization of numerical methods and probabilistic methods. Identification of faces or fingerprints need modeling and each model of the pattern is built by a choice of multi-dimensional probability distribution function and feature combination. PFC modeling via nodes combination and parameter $\gamma$ as $\mathrm{N}$-dimensional probability distribution function enables data parameterization and interpolation for feature vectors. Multi-dimensional data is modeled and interpolated via nodes combination and different functions as probability distribution functions for each feature treated as random variable: polynomial, sine, cosine, tangent, cotangent, logarithm, exponent, arc sin, arc cos, arc tan, arc cot or power function.
\end{abstract}

Keywords: image retrieval, pattern recognition, data modeling, vector interpolation, pfc method, feature reconstruction, probabilistic modeling
Volume I Issue 3 - 2017

\author{
Dariusz Jacek Jakóbczak \\ Department of Electronics and Computer Science, Koszalin \\ University of Technology, Poland
}

\begin{abstract}
Correspondence: Dariusz Jacek Jakóbczak, Department of Electronics and Computer Science, Koszalin University of Technology, Sniadeckich 2, 75-453 Koszalin, Poland,
\end{abstract} Email dariusz.jakobczak@tu.koszalin.pl

Received: October 23, 2017 | Published: October 30, 2017

\section{Introduction}

The problem of multidimensional data modeling appears in many branches of science and industry. Image retrieval, data reconstruction; object identification or pattern recognition is still the open problems in artificial intelligence and computer vision. The chapter is dealing with these questions via modeling of high-dimensional data for applications of image segmentation in image retrieval and recognition tasks. Handwriting based author recognition offers a huge number of significant implementations which make it an important research area in pattern recognition. There are so many possibilities and applications of the recognition algorithms that implemented methods have to be concerned on a single problem: retrieval, identification, verification or recognition. This chapter is concerned with two parts: image retrieval and recognition tasks. Image retrieval is based on probabilistic modeling of unknown features via combination of $\mathrm{N}$-dimensional probability distribution function for each feature treated as random variable. Handwriting and signature recognition and identification represent a significant problem. In the case of biometric writer recognition, each person is represented by the set of modeled letters or symbols. The sketch of proposed Probabilistic Features Combination (PFC) method consists of three steps: first handwritten letter or symbol must be modeled by a vector of features ( $N$-dimensional data), then compared with unknown letter and finally there is a decision of identification. Author recognition of handwriting and signature is based on the choice of feature vectors and modeling functions. So high-dimensional data interpolation in handwriting identification ${ }^{1}$ is not only a pure mathematical problem but important task in pattern recognition and artificial intelligence such as: biometric recognition, personalized handwriting recognition, ${ }^{2-4}$ automatic forensic document examination, ${ }^{5,6}$ classification of ancient manuscripts. ${ }^{7}$ Also writer recognition ${ }^{8}$ in monolingual handwritten texts is an extensive area of study and the methods independent from the language are wellseen. ${ }^{9-12}$ Proposed method represents language-independent and textindependent approach because it identifies the author via a single letter or symbol from the sample. Writer recognition methods in the recent years are going to various directions ${ }^{13-17}$ writer recognition using multiscript handwritten texts, introduction of new features, combining different types of features, studying the sensitivity of character size on writer identification, investigating writer identification in multi-script environments, impact of ruling lines on writer identification, model perturbed handwriting, methods based on run-length features, the edge-direction and edge-hinge features, a combination of codebook and visual features extracted from chain code and polygon zed representation of contours, the autoregressive coefficients, codebook and efficient code extraction methods, texture analysis with Gabor filters and extracting features, using Hidden Markov Model ${ }^{18}$ or Gaussian Mixture Model. ${ }^{19}$ So hybrid soft computing is essential: no method is dealing with writer identification via $\mathrm{N}$-dimensional data modeling or interpolation and multidimensional points comparing as it is presented in this paper. The paper wants to approach a problem of curve interpolation and shape modeling by characteristic points in handwriting identification. ${ }^{20}$ Proposed method relies on nodes combination and functional modeling of curve points situated between the basic set of key points. The functions that are used in calculations represent whole family of elementary functions with inverse functions: polynomials, trigonometric, cyclometric, logarithmic, exponential and power function. These functions are treated as probability distribution functions in the range $[0 ; 1]$. Nowadays methods apply mainly polynomial functions, for example Bernstein polynomials in Bezier curves, splines ${ }^{21}$ and NURBS. But Bezier curves don't represent the interpolation method and cannot be used for example in signature and handwriting modeling with characteristic points (nodes). Numerical methods $^{22-24}$ for data interpolation are based on polynomial or trigonometric functions, for example Lagrange, Newton, Aitken and Hermite methods. These methods have some weak sides and are not sufficient for curve interpolation in the situations when the curve cannot be build by polynomials or trigonometric functions. ${ }^{25}$ This chapter presents novel Probabilistic Features Combination (PFC) method of high-dimensional interpolation in hybrid soft computing and takes up PFC method of multidimensional data modeling. The method of PFC requires information about data (image, object, 
and curve) as the set of $\mathrm{N}$-dimensional feature vectors. Proposed PFC method is applied in image retrieval and recognition tasks via different coefficients for each feature as random variable: polynomial, sinusoidal, cosinusoidal, tangent, cotangent, logarithmic, exponential, arc sin, and arc cos, arc tan, arc cot or power. Modeling functions for PFC calculations are chosen individually for every task and they represent probability distribution functions of random variable $\alpha_{i} \varepsilon[0 ; 1]$ for every feature $i=1,2, \ldots N-1$. So this chapter wants to answer the question: how to retrieve the image using $\mathrm{N}$-dimensional feature vectors and to recognize a handwritten letter or symbol by a set of high-dimensional nodes via hybrid soft computing?

\section{Hybrid multidimensional modeling of feature vectors}

The method of PFC is computing (interpolating) unknown (unclear, noised or destroyed) values of features between two successive nodes (N-dimensional vectors of features) using hybridization of probabilistic methods and numerical methods. Calculated values (unknown or noised features such as coordinates, colors, textures or any coefficients of pixels, voxels and doxels or image parameters) are interpolated and parameterized for real number $x(c)$ or $z(c) \quad i=1,2, \ldots N-1$ between two successive values of feature. PFC method uses the combinations of nodes (N-dimensional

feature vectors) $p_{1}=\left(x_{1}, y_{1}, \ldots, z_{1}\right), \quad p_{2}=\left(x_{2}, y_{2}, \ldots, z_{2}\right)$

,$\quad p_{n}=\left(x_{n}, y_{n}, \ldots z_{n}\right)$ as $h\left(p_{1}, p_{2}, \ldots, p_{m}\right)$ and $m=1,2, \ldots n$ to interpolate unknown value of feature (for example $y$ ) for the rest of coordinates:

$$
\begin{gathered}
c_{1}=a_{1} \times x_{k}+\left(1-a_{1}\right) \times x_{k+1}, \ldots \ldots c_{N-1}=a_{N-1} \times z_{k}+\left(1-a_{N-1}\right) \times z_{k+1}, k=1,2, \ldots n-1, \\
c=\left(c_{1}, \ldots, c_{N-1}\right), \alpha=\left(\alpha_{1}, \ldots, a_{N-1}\right), \gamma_{i}=F_{i}\left(\alpha_{i}\right) \varepsilon[0 ; 1], i=1,2, \ldots N-1
\end{gathered}
$$

$$
\begin{aligned}
& y(c)=\gamma \cdot y_{k}+(1-\gamma) y_{k+1}+\gamma(1-\gamma) \cdot h\left(p_{1}, p_{2}, \ldots, p_{m}\right), \\
& \alpha_{i} \varepsilon[0 ; 1], \gamma=F(\alpha)=F\left(\alpha_{1}, \ldots, a_{N-1}\right) \varepsilon[0 ; 1] .
\end{aligned}
$$

Then $N-1$ features $c_{1}, \ldots, c_{N-1}$ are parameterized by $\alpha_{1}, \ldots, \alpha_{N-1}$ between two nodes and the last feature (for example y) is interpolated via formula (1). Of course there can be calculated

$x(c)$ or $z(c)$ using (1). Two examples of $h$ (when $N=2$ ) computed for MHR method ${ }^{26}$ with good features because of orthogonal rows and columns at Hurwitz-Radon family of matrices:

$$
h\left(p_{1}, p_{2}\right)=\frac{y_{1}}{x_{1}} x_{2}+\frac{y_{2}}{x_{2}} x_{1}
$$

or

$$
h\left(p_{1}, p_{2}, p_{3}, p_{4}\right)=\frac{1}{x_{1}{ }^{2}+x_{3}{ }^{2}}\left(x_{1} x_{2} y_{1}+x_{2} x_{3} y_{3}+x_{3} x_{4} y_{1}-x_{1} x_{4} y_{3}\right)+\frac{1}{x_{2}{ }^{2}+x_{4}{ }^{2}}\left(x_{1} x_{2} y_{2}+x_{1} x_{4} y_{4}+x_{3} x_{4} y_{2}-x_{2} x_{3} y_{4}\right) \text {. }
$$

The simplest nodes combination is

$h\left(p_{1}, p_{2}, \ldots, p_{m}\right)=0$ (3)

and then there is a formula of interpolation:

$y(c)=\gamma \cdot y_{i}+(1-\gamma) y_{i+1}$.

Formula (1) gives the infinite number of calculations for unknown feature (determined by choice of $\mathrm{F}$ and $\mathrm{h}$ ) as there is the infinite number of objects to recognize or the infinite number of images to rerieve. Nodes combination is the individual feature of each modeled data. Coefficient $\gamma=F(\alpha)$ and nodes combination $\mathrm{h}$ are key factors in PFC data interpolation and object modeling.

\section{$\mathbf{N}$-dimensional probability distribution functions in PFC modeling}

Unknown values of features, settled between the nodes, are computed using PFC method as in (1). Key question is dealing with coefficient $\gamma$. The simplest way of PFC calculation means $h=0$ and $\gamma_{i}=\alpha_{i}$ (basic probability distribution for each random variable $\alpha_{i}$ ). Then PFC represents a linear interpolation. Figure 1 is the example of curve (data) modeling when the formula is known: $y=2^{x}$. MHR method $^{26}$ is the example of PFC modeling for feature vector of dimension $N=2$. Each interpolation requires specific distributions of random variables $\alpha_{i}$ and $\gamma$ in (1) depends on parameters $\alpha_{i} \varepsilon[0 ; 1]$ :

$$
\begin{aligned}
& \gamma=F(\alpha), F:[0 ; 1]^{N-1} \rightarrow[0 ; 1], F(0, \ldots, 0)=0, \\
& F(1, \ldots, 1)=1
\end{aligned}
$$

and $F$ is strictly monotonic for each random variable $\alpha_{i}$ separately. Coefficient $\gamma_{i}$ are calculated using appropriate function and choice of function is connected with initial requirements and data specifications. Different values of coefficients $\gamma_{i}$ are connected with applied functions $F_{i}\left(\alpha_{i}\right)$. These functions $\gamma_{i}=F_{i}\left(\alpha_{i}\right)$ represent the examples of probability distribution functions for random variable $\alpha_{i} \varepsilon[0 ; 1]$ and real number $s>0, i=1,2, \ldots N-1$ :

$$
\begin{aligned}
& \gamma_{i}=\alpha_{i}^{s}, \gamma_{i}=\sin \left(\alpha_{i}^{s} \cdot \pi / 2\right), \gamma_{i}=\sin ^{s}\left(\alpha_{i} \cdot \pi / 2\right), \\
& \gamma_{i}=1-\cos \left(\alpha_{i}^{s} \cdot \pi / 2\right), \gamma_{i}=1-\cos ^{s}\left(\alpha_{i} \cdot \pi / 2\right),
\end{aligned}
$$




$$
\begin{gathered}
\gamma_{i}=\tan \left(\alpha_{i}^{s} \cdot \pi / 4\right), \gamma_{i}=\tan ^{s}\left(\alpha_{i} \cdot \pi / 4\right), \gamma_{i}=\log _{2}\left(\alpha_{i}^{s}+1\right) \\
\gamma_{i}=\log _{2}{ }^{s}\left(\alpha_{i}+1\right), \gamma_{i}=\left(2^{\alpha}-1\right)^{s}, \gamma_{i}=2 / \pi \cdot \arcsin \left(\alpha_{i}^{s}\right), \\
\gamma_{i}=\left(2 / \pi \cdot \arcsin \alpha_{i}\right)^{s}, \gamma_{i}=1-2 / \pi \cdot \arccos \left(\alpha_{i}^{s}\right), \\
\gamma_{i}=1-\left(2 / \pi \cdot \arccos \alpha_{i}\right)^{s}, \gamma_{i}=4 / \pi \cdot \arctan \left(\alpha_{i}^{s}\right), \\
\gamma_{i}=\left(4 / \pi \cdot \arctan \alpha_{i}\right)^{s}, \gamma_{i}=\operatorname{ctg}\left(\pi / 2-\alpha_{i}^{s} \cdot \pi / 4\right), \\
\gamma_{i}=\operatorname{ctg}^{s}\left(\pi / 2-\alpha_{i} \cdot \pi / 4\right), \gamma_{i}=2-4 / \pi \cdot \operatorname{arcctg}\left(\alpha_{i}^{s}\right), \gamma_{i}=\left(2-4 / \pi \cdot \operatorname{arcctg} \alpha_{i}\right)^{s}
\end{gathered}
$$

or any strictly monotonic function between points $(0 ; 0)$ and $(1 ; 1)$ - for example combinations of these functions. Interpolations of function $y=2^{x}$ for $N=2, h=0$ and $\gamma=\alpha^{s}$ with $s=0.8$

(Figure 2) or $\gamma=\log _{2}(\alpha+1)$ (Figure 3) are quite better then linear interpolation (Figure 1).

Functions $\gamma_{i}$ are strictly monotonic for each random variable $\alpha_{i} \varepsilon[0 ; 1]$ as $\gamma=F(\alpha)$ is $N$-dimensional probability distribution function, for example:

$$
\gamma=\frac{1}{N-1} \sum_{i=1}^{N-1} \gamma_{i}, \gamma=\prod_{i=1}^{N-1} \gamma_{i}
$$

and every monotonic combination of $\gamma_{i}$ such as

$$
\gamma=F(\alpha), F:[0 ; 1]^{N-1} \rightarrow[0 ; 1], F(0, \ldots, 0)=0, F(1, \ldots, 1)=1
$$

For example when $N=3$ there is a bilinear interpolation:

$$
\gamma_{1}=\alpha_{1}, \gamma_{2}=\alpha_{2}, \gamma=1 / 2\left(\alpha_{1}+\alpha_{2}\right)
$$

or a bi-quadratic interpolation:

$$
\gamma_{1}=\alpha_{1}^{2}, \gamma_{2}=\alpha_{2}^{2}, \gamma=1 / 2\left(\alpha_{1}^{2}+\alpha_{2}^{2}\right)
$$

or a bi-cubic interpolation:

$$
\gamma_{1}=\alpha_{1}^{3}, \gamma_{2}=\alpha_{2}^{3}, \gamma=1 / 2\left(\alpha_{1}^{3}+\alpha_{2}^{3}\right)
$$

or others modeling functions $\gamma$. Choice of functions $\gamma_{i}$ and value $s$ depends on the specifications of feature vectors and individual requirements. What is very important in PFC method: two data sets (for example a handwritten letter or signature) may have the same set of nodes (feature vectors: pixel coordinates, pressure, speed, angles) but different $h$ or $\gamma$ results in different interpolations (Figures 4-6). Here are three examples of PFC reconstruction (Figures 4-6) for $N=2$ and four nodes: $(-1.5 ;-1),(1.25 ; 3.15),(4.4 ; 6.8)$ and $(8 ; 7)$. Formula of the curve is not given. Algorithm of PFC retrieval, interpolation and modeling consists of five steps: first choice of nodes $p_{i}$ (feature vectors), then choice of nodes combination $h\left(p_{1}, p_{2}, \ldots, p_{m}\right)$, choice of distribution (modeling function) $\gamma=F(\alpha)$, determining values of $\alpha_{i} \varepsilon[0 ; 1]$ and finally the computations (1).

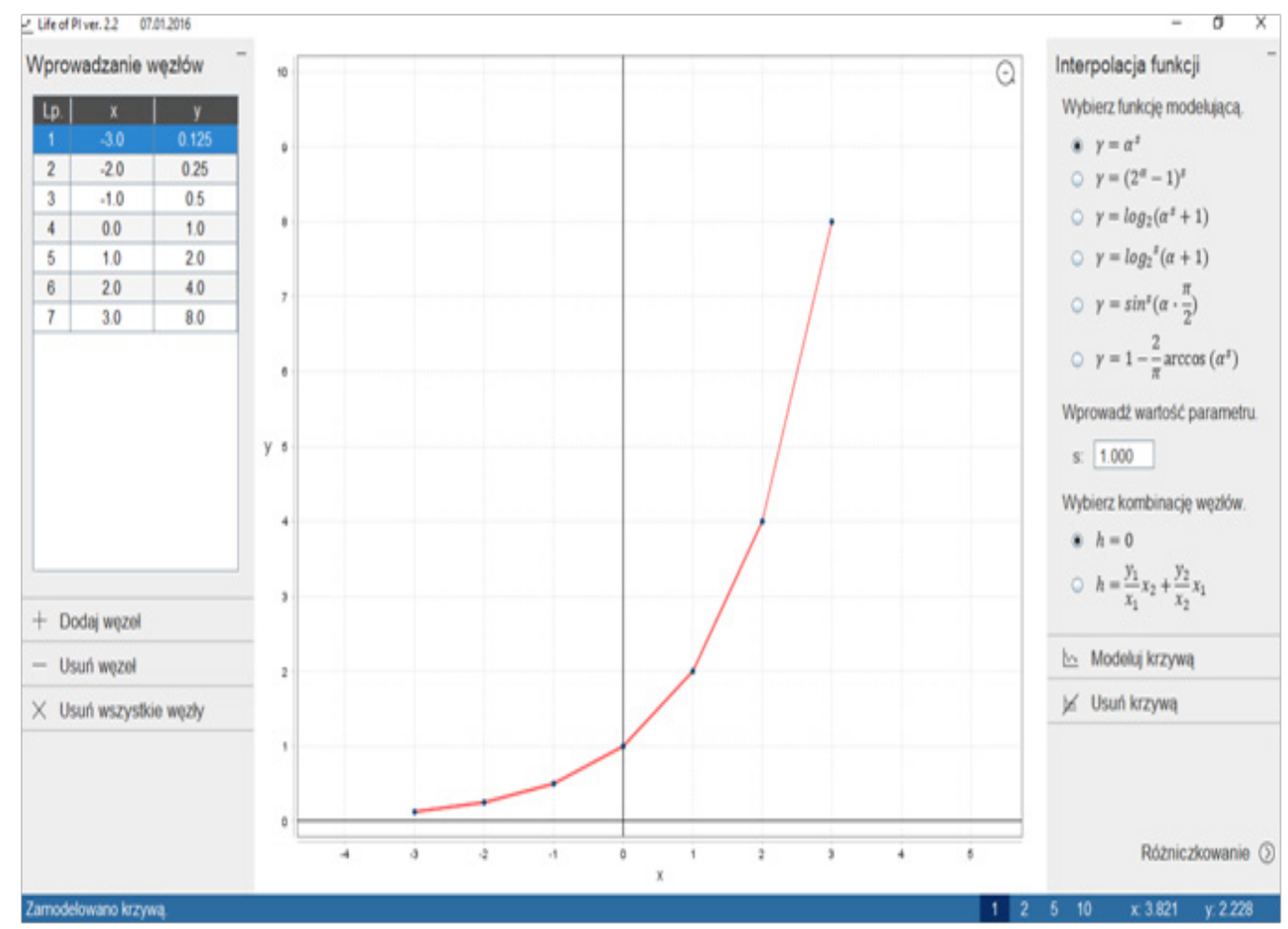

Figure I PFC linear 2D modeling of function $y=2^{x}$ with seven nodes (in left window) and options in right window (modeling functions $\gamma$ and nodes combination $h$ ). 


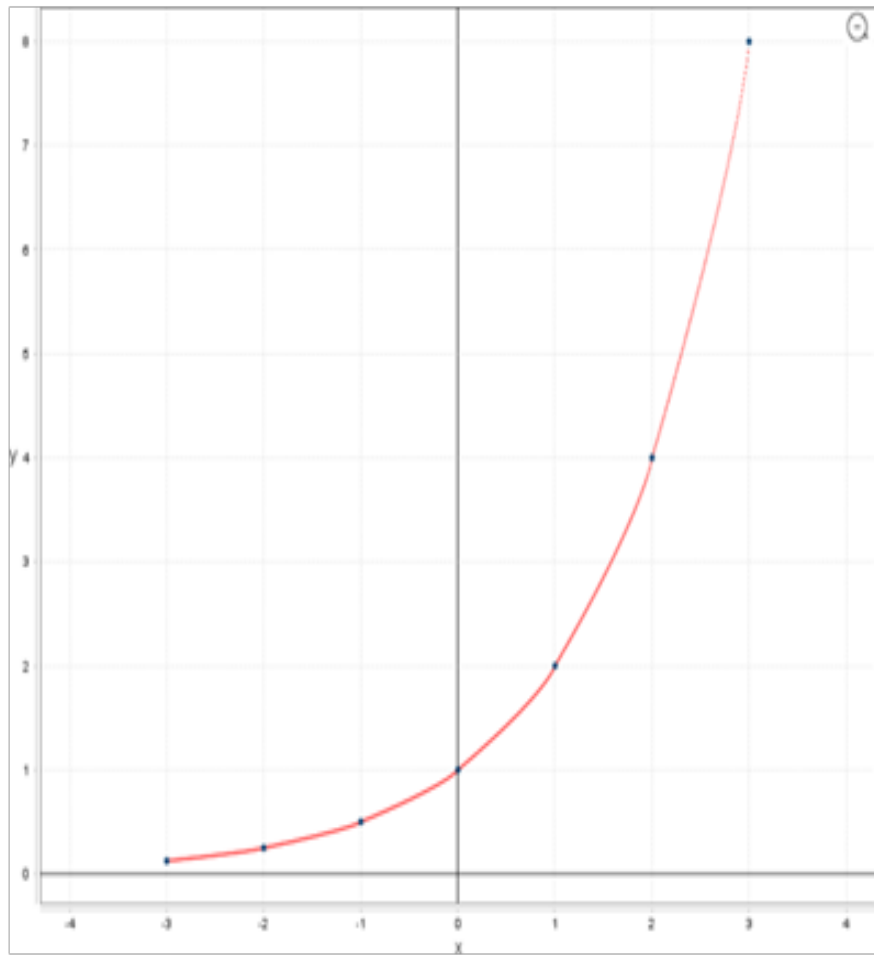

Figure 2 PFC two-dimensional modeling of function $y=2^{x}$ with seven nodes as Figure I and $h=0, \gamma=\alpha^{0.8}$.

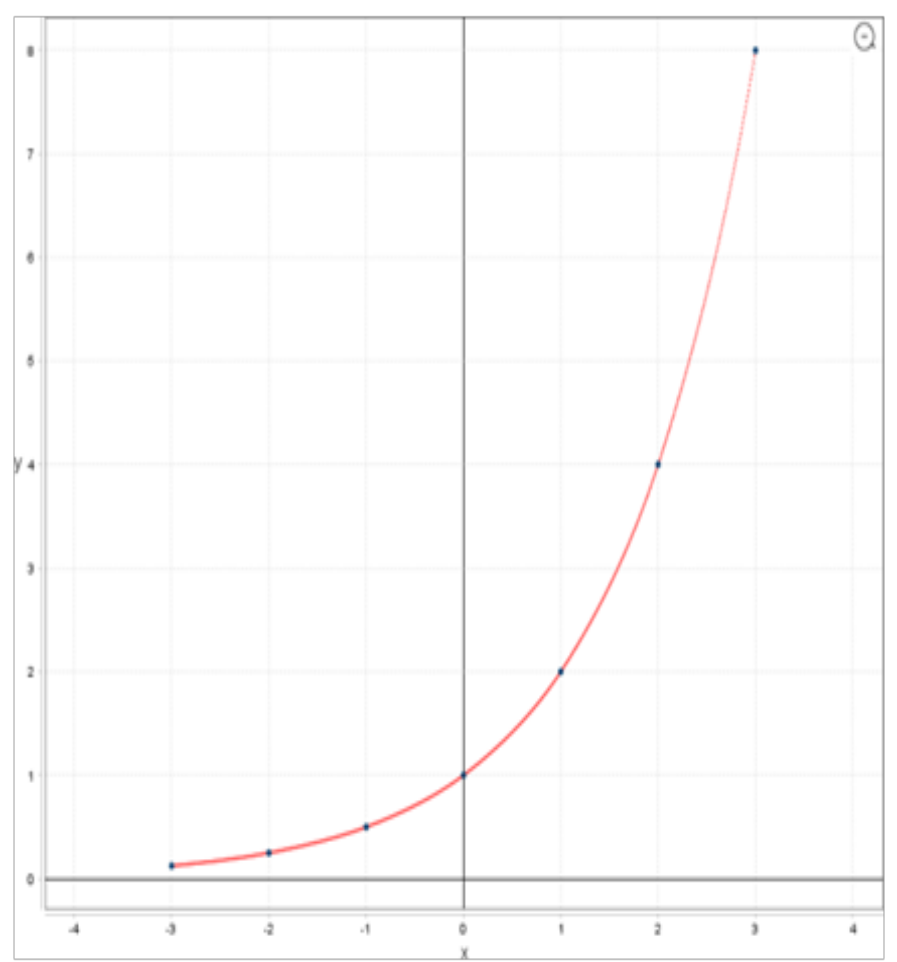

Figure 3 PFC two-dimensional reconstruction of function $y=2^{x}$ with seven nodes as Figure I and $h=0, \gamma=\log _{2}(\alpha+1)$.

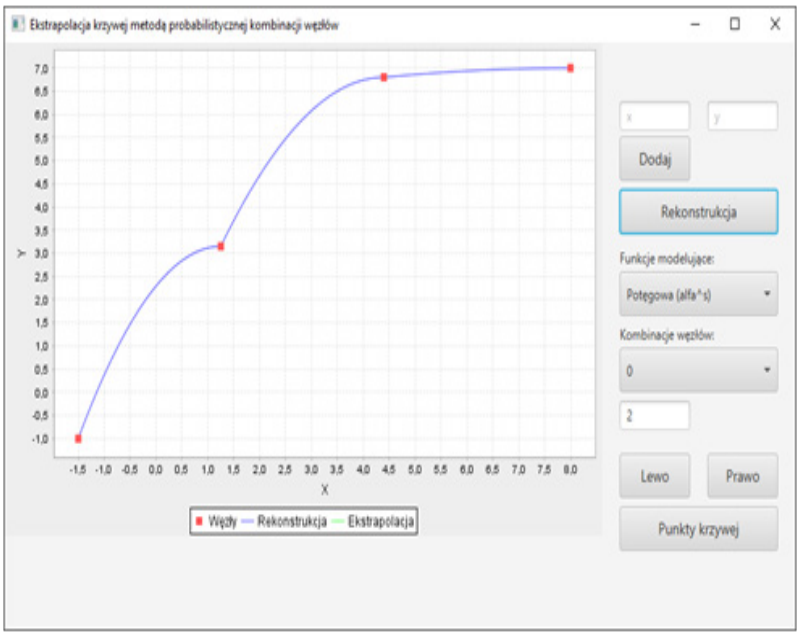

Figure 4 PFC 2D modeling for $\gamma=\alpha^{2}$ and $h=0$.

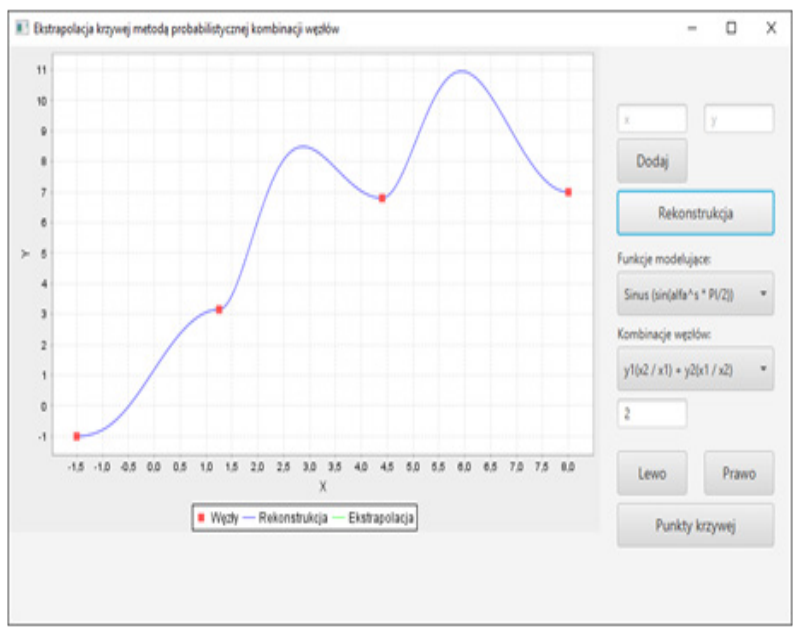

Figure 5 PFC 2D reconstruction for $\gamma=\sin \left(\alpha^{2} \cdot \pi / 2\right)$ and $h$ in (2).

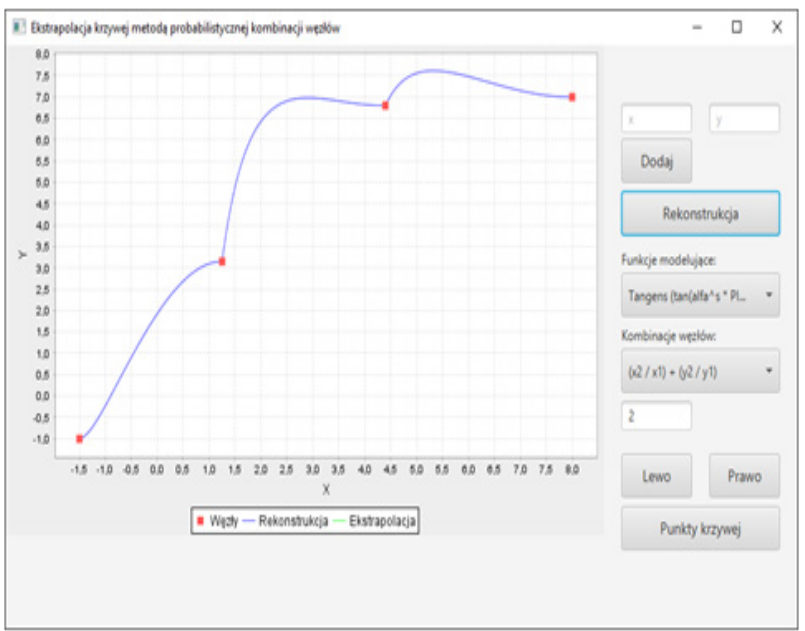

Figure 6 PFC 2D interpolation for $\gamma=\tan \left(\alpha^{2} \cdot \pi / 4\right)$ and $h=\left(x_{2} / x_{1}\right)+\left(y_{2} / y_{1}\right)$. 


\section{Image retrieval via PFC high-dimensional feature reconstruction}

After the process of image segmentation and during the next steps of retrieval, recognition or identification, there is a huge number of features included in N-dimensional feature vector. These vectors can be treated as "points" in N-dimensional feature space. For example in artificial intelligence there is a high-dimensional search space (the set of states that can be reached in a search problem) or hypothesis space (the set of hypothesis that can be generated by a machine learning algorithm). This paper is dealing with multidimensional feature spaces that are used in computer vision, image processing and machine learning. Having monochromatic (binary) image which consists of some objects, there is only 2-dimensional feature $\operatorname{space}\left(x_{i}, y_{i}\right)$ coordinates of black pixels or coordinates of white pixels. No other parameters are needed. Thus any object can be described by a contour (closed binary curve). Binary images are attractive in processing (fast and easy) but don't include important information. If the image has grey shades, there is 3 -dimensional feature space $\left(x_{i}, y_{i}, z_{i}\right)$ with grey shade $z_{i}$. For example most of medical images are written in grey shades to get quite fast processing. But when there are color images (three parameters for RGB or other color systems) with textures or medical data or some parameters, then it is N-dimensional feature space. Dealing with the problem of classification learning for high-dimensional feature spaces in artificial intelligence and machine learning (for example text classification and recognition), there are some methods: decision trees, $k$-nearest neighbors, perceptrons, naïve Bayes or neural networks methods. All of these methods are struggling with the curse of dimensionality: the problem of having too many features. And there are many approaches to get less number of features and to reduce the dimension of feature space for faster and less expensive calculations. This paper aims at inverse problem to the curse of dimensionality: dimension $N$ of feature space (i.e. number of features) is unchanged, but number of feature vectors (i.e. "points" in $\mathrm{N}$-dimensional feature space) is reduced into the set of nodes. So the main problem is as follows: how to fix the set of feature vectors for the image and how to retrieve the features between the "nodes"? This paper aims in giving the answer of this question.

\section{Grey scale image retrieval using PFC 3D method}

Binary images are just the case of 2D points $(x, y): 0$ or 1 , black or white, so retrieval of monochromatic images is done for the closed curves (first and last node are the same) as the contours of the objects for $N=2$ and examples as (Figure 1-6). Grey scale images are the case of 3D points $(x, y, s)$ with $s$ as the shade of grey. So the grey scale between the nodes $p_{1}=\left(x_{1}, y_{1}, s_{1}\right)$ and $p_{2}=\left(x_{2}, y_{2}, s_{2}\right)$ is computed with $\gamma=F(\alpha)=F\left(\alpha_{1}, \alpha_{2}\right)$ as (1) and for example

(4)-(6) or others modeling functions $\gamma_{i}$. As the simple example two successive nodes of the image are: left upper corner with coordinates $p_{1}=\left(x_{1}, y_{1}, 2\right)$ and right down corner $p_{2}=\left(x_{2}, y_{2}, 10\right)$. The image retrieval with the grey scales 2-10 between $p_{1}$ and $p_{2}$ looks as follows for a bilinear interpolation (4) (Figure 7) (Figure 8).

\begin{tabular}{|l|l|l|l|l|l|l|l|l|}
\hline 2 & 3 & 4 & 5 & 6 & 7 & 8 & 9 & 10 \\
\hline 2 & 3 & 4 & 5 & 6 & 7 & 8 & 9 & 10 \\
\hline 2 & 3 & 4 & 5 & 6 & 7 & 8 & 9 & 10 \\
\hline 2 & 3 & 4 & 5 & 6 & 7 & 8 & 9 & 10 \\
\hline 2 & 3 & 4 & 5 & 6 & 7 & 8 & 9 & 10 \\
\hline 2 & 3 & 4 & 5 & 6 & 7 & 8 & 9 & 10 \\
\hline 2 & 3 & 4 & 5 & 6 & 7 & 8 & 9 & 10 \\
\hline 2 & 3 & 4 & 5 & 6 & 7 & 8 & 9 & 10 \\
\hline 2 & 3 & 4 & 5 & 6 & 7 & 8 & 9 & 10 \\
\hline
\end{tabular}

Figure 7 Reconstructed grey scale numbered at each pixel.

\begin{tabular}{|l|l|l|l|l|l|l|l|l|}
\hline 2 & 2 & 2 & 2 & 2 & 2 & 2 & 2 & 2 \\
\hline 2 & 3 & 3 & 3 & 3 & 3 & 3 & 3 & 3 \\
\hline 2 & 3 & 4 & 4 & 4 & 4 & 4 & 4 & 4 \\
\hline 2 & 3 & 4 & 5 & 5 & 5 & 5 & 5 & 5 \\
\hline 2 & 3 & 4 & 5 & 6 & 6 & 6 & 6 & 6 \\
\hline 2 & 3 & 4 & 5 & 6 & 7 & 7 & 7 & 7 \\
\hline 2 & 3 & 4 & 5 & 6 & 7 & 8 & 8 & 8 \\
\hline 2 & 3 & 4 & 5 & 6 & 7 & 8 & 9 & 9 \\
\hline 2 & 3 & 4 & 5 & 6 & 7 & 8 & 9 & 10 \\
\hline
\end{tabular}

Figure 8 Grey scale image with shades of grey retrieved at each pixel. The feature vector of dimension $N=3$ is called a voxel.

\section{Color image retrieval via PFC method}

Color images in for example RGB color system $(r, g, b)$ are the set of points $(x, y, r, g, b)$ in a feature space of dimension $N=5$ . There can be more features, for example texture $t$, and then one pixel $(x, y, r, g, b, t)$ exists in a feature space of dimension $N=6$. But there are the sub-spaces of a feature space of dimension $N_{1}<N$ , for example, $(x, y, g),(x, y, b)$ or $(x, y, t)$ are points in a feature sub-space of dimension $N_{1}=3$. Reconstruction and interpolation of color coordinates or texture parameters is done like in chapter 3.1 for dimension $N=3$. Appropriate combination of $\alpha_{1}$ and $\alpha_{2}$ leads to modeling of color $r, g, b$ or texture $t$ or another feature between the nodes. And for example $(x, y, r, t),(x, y, g, t),(x, y, b, t))$ are points in a feature sub-space of dimension $N_{1}=4$ called doxels. Appropriate combination of $\alpha_{1}, \alpha_{2}$ and $\alpha_{3}$ leads to modeling of texture $t$ or another feature between the nodes. For example color image, given as the set of doxels $(x, y, r, t)$, is described for coordinates $(x, y)$ via pairs $(r, t)$ interpolated between nodes $\left(x_{1}, y_{1}, 2,1\right)$ and $\left(x_{2}, y_{2}, 10,9\right)$ as follows Figure 9 So dealing with feature space of dimension $N$ and using PFC method there is no problem called "the curse of dimensionality" and no problem called "feature selection" because 
each feature is important. There is no need to reduce the dimension $N$ and no need to establish which feature is "more important" or "less important". Every feature that depends from $N_{1}-1$ other features can be interpolated (reconstructed) in the feature sub-space of dimension $N_{1}<N$ via PFC method. But having a feature space of dimension $N$ and using PFC method there is another problem: how to reduce the number of feature vectors and how to interpolate (retrieve) the features between the known vectors (called nodes).

\begin{tabular}{|l|l|l|l|l|l|l|l|l|}
\hline 2,1 & 3,1 & 4,1 & 5,1 & 6,1 & 7,1 & 8,1 & 9,1 & 10,1 \\
\hline 2,2 & 3,2 & 4,2 & 5,2 & 6,2 & 7,2 & 8,2 & 9,2 & 10,2 \\
\hline 2,3 & 3,3 & 4,3 & 5,3 & 6,3 & 7,3 & 8,3 & 9,3 & 10,3 \\
\hline 2,4 & 3,4 & 4,4 & 5,4 & 6,4 & 7,4 & 8,4 & 9,4 & 10,4 \\
\hline 2,5 & 3,5 & 4,5 & 5,5 & 6,5 & 7,5 & 8,5 & 9,5 & 10,5 \\
\hline 2,6 & 3,6 & 4,6 & 5,6 & 6,6 & 7,6 & 8,6 & 9,6 & 10,6 \\
\hline 2,7 & 3,7 & 4,7 & 5,7 & 6,7 & 7,7 & 8,7 & 9,7 & 10,7 \\
\hline 2,8 & 3,8 & 4,8 & 5,8 & 6,8 & 7,8 & 8,8 & 9,8 & 10,8 \\
\hline 2,9 & 3,9 & 4,9 & 5,9 & 6,9 & 7,9 & 8,9 & 9,9 & 10,9 \\
\hline
\end{tabular}

Figure 9 Color image with color and texture parameters $(r, t)$ interpolated at each pixel.

\begin{tabular}{|l|l|l|l|l|l|l|l|l|}
\hline 2 & 2 & 2 & 2 & 2 & 2 & 2 & 2 & 2 \\
\hline 2 & 3 & 3 & 3 & 3 & 3 & 3 & 3 & 3 \\
\hline 2 & 3 & 4 & 4 & 4 & 4 & 4 & 4 & 4 \\
\hline 2 & 3 & 4 & 5 & 5 & 5 & 5 & 5 & 5 \\
\hline 2 & 3 & 4 & 5 & 6 & 6 & 6 & 6 & 6 \\
\hline 2 & 3 & 4 & 5 & 6 & 7 & 7 & 7 & 7 \\
\hline 2 & 3 & 4 & 5 & 6 & 7 & 8 & 8 & 8 \\
\hline 2 & 3 & 4 & 5 & 6 & 7 & 8 & 9 & 9 \\
\hline 2 & 3 & 4 & 5 & 6 & 7 & 8 & 9 & 10 \\
\hline
\end{tabular}

Figure 10 The curse of dimensionality with feature selection wants to eliminate some rows from the feature matrix and to reduce dimension $\mathrm{N}$.

\begin{tabular}{|l|l|l|l|l|l|l|l|l|}
\hline 2 & 2 & 2 & 2 & 2 & 2 & 2 & 2 & 2 \\
\hline 2 & 3 & 3 & 3 & 3 & 3 & 3 & 3 & 3 \\
\hline 2 & 3 & 4 & 4 & 4 & 4 & 4 & 4 & 4 \\
\hline 2 & 3 & 4 & 5 & 5 & 5 & 5 & 5 & 5 \\
\hline 2 & 3 & 4 & 5 & 6 & 6 & 6 & 6 & 6 \\
\hline 2 & 3 & 4 & 5 & 6 & 7 & 7 & 7 & 7 \\
\hline 2 & 3 & 4 & 5 & 6 & 7 & 8 & 8 & 8 \\
\hline 2 & 3 & 4 & 5 & 6 & 7 & 8 & 9 & 9 \\
\hline 2 & 3 & 4 & 5 & 6 & 7 & 8 & 9 & 10 \\
\hline
\end{tabular}

\begin{tabular}{|l|l|l|l|l|l|l|}
\hline 2 & 2 & 2 & 2 & 2 & 2 & 2 \\
\hline 2 & 3 & 3 & 3 & 3 & 3 & 3 \\
\hline 2 & 3 & 4 & 4 & 4 & 4 & 4 \\
\hline 2 & 3 & 4 & 5 & 5 & 5 & 5 \\
\hline 2 & 3 & 4 & 5 & 6 & 6 & 6 \\
\hline 2 & 3 & 4 & 5 & 6 & 7 & 7 \\
\hline 2 & 3 & 4 & 5 & 6 & 7 & 8 \\
\hline 2 & 3 & 4 & 5 & 6 & 7 & 8 \\
\hline 2 & 3 & 4 & 5 & 6 & 7 & 8 \\
\hline
\end{tabular}

Figure II PFC method wants to eliminate some columns from the feature matrix and to reduce dimension M. 


\section{Recognition tasks via high-dimensional feature vectors' interpolation}

The process of biometric recognition and identification consists of three parts: pre-processing, image segmentation with feature extraction and recognition or verification. Pre-processing is a common stage for all methods with binarization, thinning, size standardization. Proposed online approach is based on 2D curve modeling and multidimensional feature vectors' interpolation. Feature extraction gives the key points (nodes as $N$-dimensional feature vectors) that are used in PFC curve reconstruction and identification. PFC method enables signature and handwriting recognition, which is used for biometric purposes, because human signature or handwriting consists of nontypical curves and irregular shapes (for example (Figures 4-6). The language does not matter because each symbol is treated as a curve. This process of recognition consists of three parts:

A. Before recognition: continual and never-ending building the data basis: patterns' modeling - choice of nodes combination, probabilistic distribution function (1) and values of features (pen pressure, speed, pen angle etc.) appearing in high dimensional feature vectors for known signature or handwritten letters of some persons in the basis.

Feature extraction: unknown author - fixing the values in feature vectors for unknown signature or handwritten words: $N$-dimensional feature vectors $(x, y, p, s, a, t)$ with $\mathrm{x}, \mathrm{y}$-points' coordinates, $p$-pen pressure, $s$-speed of writing, $a$ - pen angle or any other features $t$.

The result: recognition or identification - comparing the results of PFC interpolation for known patterns from the data basis with features of unknown object.

\section{Signature modeling and multidimensional recognition}

Human signature or handwriting consists mainly of non-typical curves and irregular shapes. So how to model two-dimensional handwritten characters via PFC method? Each model has to be described (1) by the set of nodes, nodes combination $h$ and a function $\gamma=F(\alpha)$ for each letter. Other features in multi-dimensional feature space are not visible but used in recognition process (for example $p$-pen pressure, $s$-speed of writing, $a$ - pen angle). Less complicated models can take $h\left(p_{1}, p_{2}, \ldots, p_{m}\right)=0$ and then the formula of interpolation (1) looks as follows:

$$
y(c)=\gamma \cdot y_{i}+(1-\gamma) y_{i+1} .
$$

Formula (7) represents the simplest linear interpolation for basic probability distribution if $\gamma=\alpha$. Here are some examples of nontypical curves and irregular shapes as the whole signature or a part of signature, reconstructed via PFC method for seven nodes $(x, y)$ (Figures 1-3).

Figures 12-18 are two-dimensional subspace of $N$-dimensional feature space, for example $(x, y, p, s, a, t)$ when $N=6$. If the recognition process is working "offline" and features $p$-pen pressure, $s$-speed of writing, $a$-pen angle or another feature $t$ are not given, the only information before recognition is situated in $x, y$-points' coordinates. After pre-processing (binarization, thinning, size standarization), feature extraction is second part of biometric identification. Choice of characteristic points (nodes) for unknown letter or handwritten symbol is a crucial factor in object recognition. The range of coefficients $x$ has to be the same like the $x$ range in the basis of patterns. When the nodes are fixed, each coordinate of every chosen point on the curve $\left(x_{0}{ }^{c}, y_{0}{ }^{c}\right),\left(x_{1}{ }^{c}, y_{1}{ }^{c}\right), \ldots,\left(x_{M}{ }^{c}, y_{M}{ }^{c}\right)$ is accessible to be used for comparing with the models. Then probability distribution function $\gamma=F(\alpha)$ and nodes combination $h$ have to be taken from the basis of modeled letters to calculate appropriate second coordinates $y_{i}^{(j)}$ of the pattern for first coordinates $x_{i}{ }^{c}, i=0,1, \ldots, M$. After interpolation it is possible to compare given handwritten symbol with a letter in the basis of patterns. Comparing the results of PFC interpolation for required second coordinates of a model in the basis of patterns with points on the curve $\left(x_{0}{ }^{c}, y_{0}{ }^{c}\right),\left(x_{1}{ }^{c}, y_{1}{ }^{c}\right), \ldots,\left(x_{M}{ }^{c}, y_{M}{ }^{c}\right)$ , one can say if the letter or symbol is written by person P1, P2 or another. The comparison and decision of recognition [26] is done via minimal distance criterion. Curve points of unknown handwritten symbol are: $\left(x_{0}{ }^{c}, y_{0}{ }^{c}\right),\left(x_{1}{ }^{c}, y_{1}{ }^{c}\right), \ldots,\left(x_{M}{ }^{c}, y_{M}{ }^{c}\right)$ .The criterion of recognition for models $S_{j}=\left\{\left(x_{0}{ }^{c}, y_{0}{ }^{(j)}\right),\left(x_{1}{ }^{c}, y_{1}{ }^{(j)}\right), \ldots,\left(x_{M}{ }^{c}, y_{M}{ }^{(j)}\right), j=0,1,2,3 \ldots K\right\}$ is given as:

$$
\sum_{i=0}^{M}\left|y_{i}^{c}-y_{i}^{(j)}\right| \rightarrow \min \text { Or } \sqrt{\sum_{i=0}^{M}\left|y_{i}^{c}-y_{i}^{(j)}\right|^{2}} \rightarrow \min .
$$

\begin{tabular}{|c|c|c|}
\hline Lp. & $x$ & $y$ \\
\hline 1 & -3.0 & 0.125 \\
\hline 2 & -2.0 & 0.25 \\
\hline 3 & -1.0 & 0.5 \\
\hline 4 & 0.0 & 1.0 \\
\hline 5 & 1.0 & 2.0 \\
\hline 6 & 2.0 & 4.0 \\
\hline 7 & 3.0 & 8.0 \\
\hline
\end{tabular}

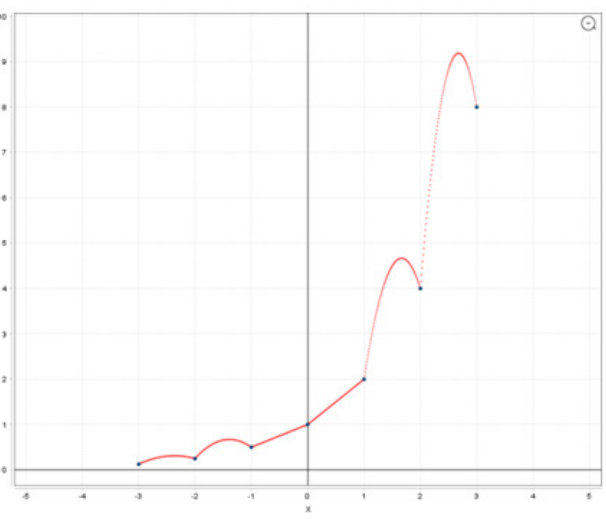

Figure I2 PFC 2D interpolation for $\gamma=\alpha^{s}, s=1, h=\frac{y_{1}}{x_{1}} x_{2}+\frac{y_{2}}{x_{2}} x_{1}$. 


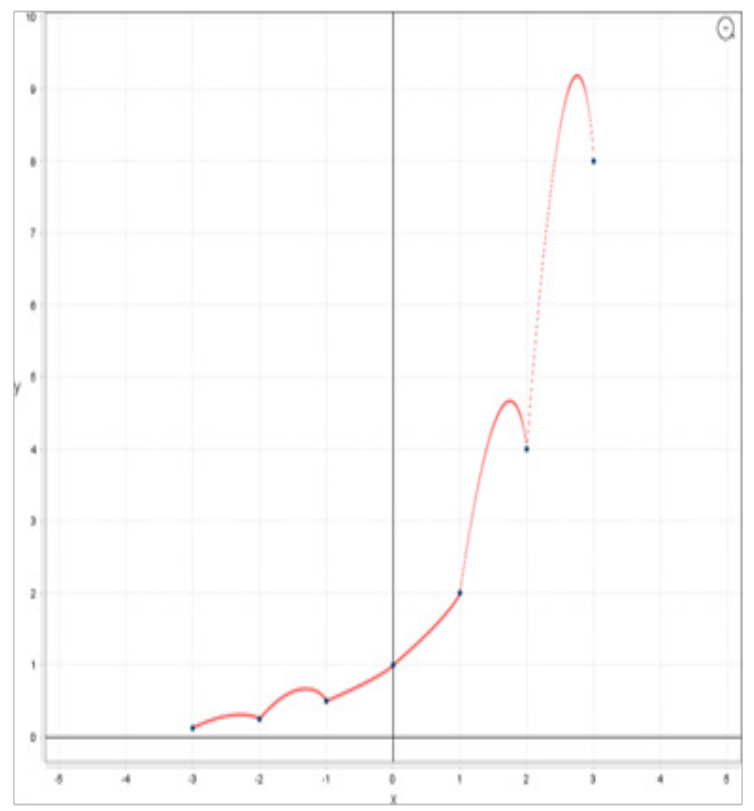

Figure I3 PFC 2D modeling for $\gamma=\alpha^{s}, s=0,8, h=\frac{y_{1}}{x_{1}} x_{2}+\frac{y_{2}}{x_{2}} x_{1}$.

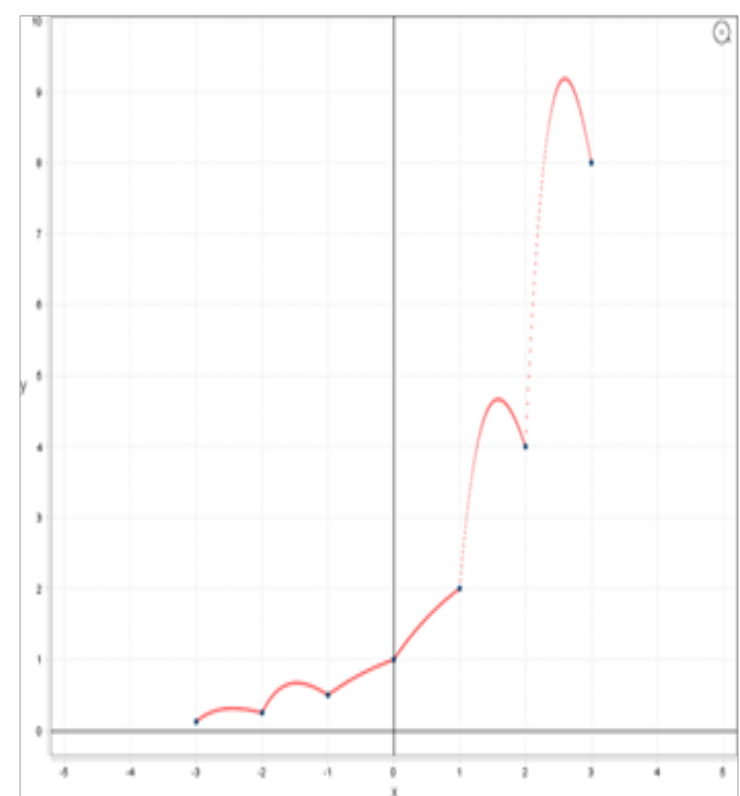

Figure I4 PFC 2D reconstruction for $\gamma=\left(2^{\alpha}-1\right)^{s}, s=1, h=\frac{y_{1}}{x_{1}} x_{2}+\frac{y_{2}}{x_{2}} x_{1}$

Minimal distance criterion helps us to fix a candidate for unknown writer as a person from the model $S_{j}$ in the basis. If the recognition process is "online" and features $p$-pen pressure, $s$-speed of writing, $a$ pen angle or some feature $t$ are given, then there is more information in the process of author recognition, identification or verification in a feature space $(x, y, p, s, a, t)$ of dimension $N=6$ or others. Some person may know how the signature of another man looks like for example (Figures 4-6) or (Figures 12-18), but other extremely important features $p, s, a, t$ are not visible. Dimension $N$ of a feature space may be very high, but this is no problem. As it is illustrated
(Figure 10) (Figure 11) the problem connected with the curse of dimensionality with feature selection does not matter. There is no need to fix which feature is less important and can be eliminated. Every feature is very important and each of them can be interpolated between the nodes using PFC high-dimensional interpolation. For example pressure of the pen $p$ differs during the signature writing and $p$ is changing for particular letters or fragments of the signature.

Then feature vector $(x, y, p)$ of dimension $N_{1}=3$ is dealing with $p$ interpolation at the point $(x, y)$ via modeling functions (4)-(6) or others. If angle of the pen $a$ differs during the signature writing and $a$ is changing for particular letters or fragments of the signature, then feature vector $(x, y, a)$ of dimension $N_{1}=3$ is dealing with $a$ interpolation at the point $(x, y)$ via modeling functions (4)-(6) or others. If speed of the writing $s$ differs during the signature writing and $s$ is changing for particular letters or fragments of the signature, then feature vector $(x, y, s)$ of dimension $N_{1}=3$ is dealing with $s$ interpolation at the point $(x, y)$ via modeling functions (4)-(6) or others. This PFC 3D interpolation is the same like in chapter 3.1 grey scale image retrieval but for selected pairs $\left(\alpha_{1}, \alpha_{2}\right)$ - only for the points of signature between $\left(x_{1}, y_{1}, 2\right)$ and $\left(x_{2}, y_{2}, 10\right)$ : If a feature sub-space is dimension $N_{1}=4$ and feature vector is for example $(x, y, p, s)$, then PFC 4D interpolation is the same like in chapter 3.2 color image retrieval but for selected pairs $\left(\alpha_{1}, \alpha_{2}\right)$ - only for the points of signature between $\left(x_{1}, y_{1}, 2,1\right)$ and $\left(x_{2}, y_{2}, 10,9\right)$ : If a feature sub-space is dimension $N_{1}=5$ and feature vector is for example $(x, y, p, s, a)$, then PFC 5D interpolation is the same like in chapter 3.2 color image retrieval but for selected pairs $\left(\alpha_{1}, \alpha_{2}\right)-$ only for the points of signature between $\left(x_{1}, y_{1}, 2,1,30\right)$ and $\left(x_{2}, y_{2}, 10,9,60\right)$ : (Figures 19-21) are the examples of denotation for the features that are not visible during the signing or handwriting but very important in the process of "online" recognition, identification or verification.

Even if from technical reason or other reasons only some points of signature or handwriting (feature nodes) are given in the process of "online" recognition, identification or verification, the values of features between nodes are computed via multidimensional PFC interpolation like for example between $\left(x_{1}, y_{1}, 2\right)$ and $\left(x_{2}, y_{2}, 10\right)$ on Figure 19, between $\left(x_{1}, y_{1}, 2,1\right)$ and $\left(x_{2}, y_{2}, 10,9\right)$ on (Figure 20) or between $\left(x_{1}, y_{1}, 2,1,30\right)$ and $\left(x_{2}, y_{2}, 10,9,60\right)$ on (Figure 21). Reconstructed features are compared with the features in the basis of patterns like parameter $y$ in (8) and appropriate criterion gives the result. So persons with the parameters of their signatures are allocated in the basis of patterns. The curve does not have to be smooth at the nodes because handwritten symbols are not smooth. The range of coefficients $x$ has to be the same for all models because of comparing appropriate coordinates $y$. Every letter or a part of signature is modeled by PFC via three factors: the set of high-dimensional feature nodes, probability distribution function $\gamma=F(\alpha)$ and nodes combination $h$. These three factors are chosen individually for each letter or a part of signature therefore this information about modeled curves seems to be enough for specific PFC multidimensional curve interpolation and handwriting identification. What is very important, $\mathrm{PFC} N$-dimensional 
modeling is independent of the language or a kind of symbol (letters, numbers, characters or others). One person may have several patterns for one handwritten letter or signature. Summarize: every person has the basis of patterns for each handwritten letter or symbol, described by the set of feature nodes, modeling function $\gamma=F(\alpha)$ and nodes combination $h$. Whole basis of patterns consists of models $S_{j}$ for $j=0,1,2,3 \ldots K$.

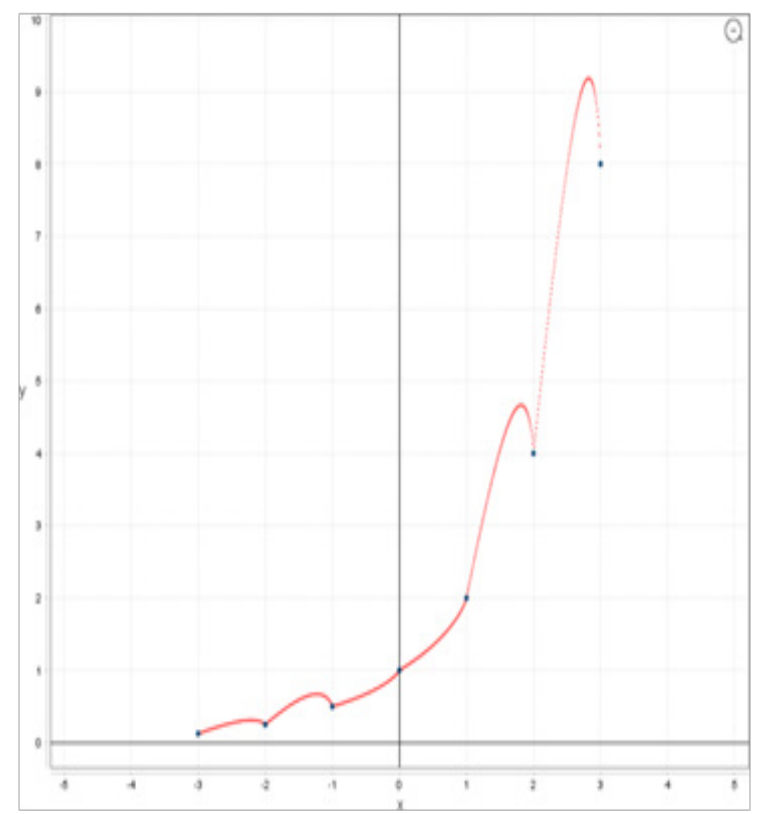

Figure 15 PFC 2D reconstruction for $\gamma=\log _{2}\left(\alpha^{s}+1\right), s=0,8, h=\frac{y_{1}}{x_{1}} x_{2}+\frac{y_{2}}{x_{2}} x_{1}$

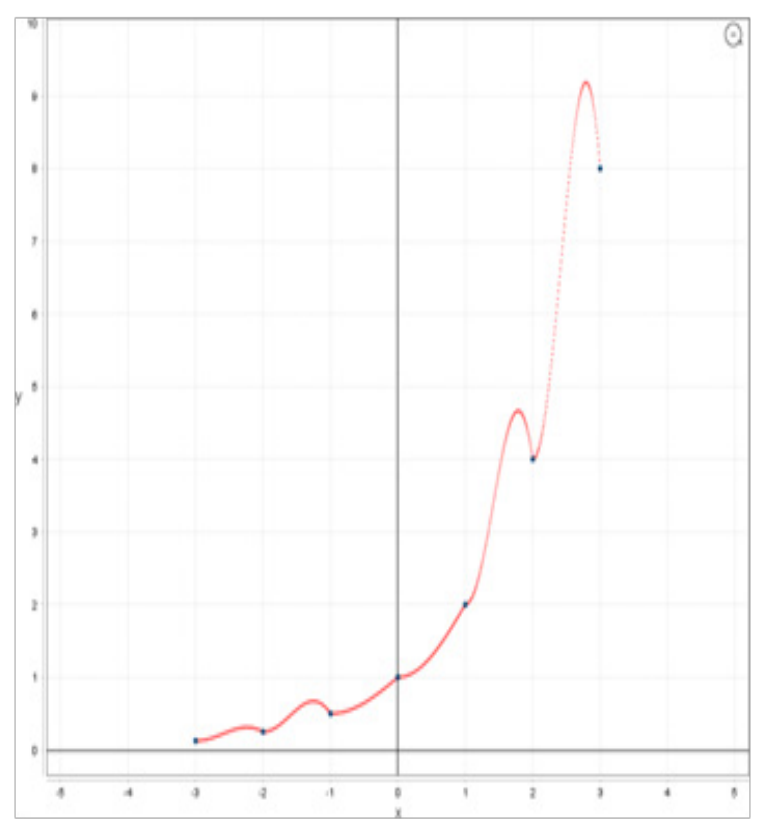

Figure 16 PFC 2D interpolation for $\gamma=\sin ^{s}\left(\alpha \cdot \frac{\pi}{2}\right), s=1, h=\frac{y_{1}}{x_{1}} x_{2}+\frac{y_{2}}{x_{2}} x_{1}$.

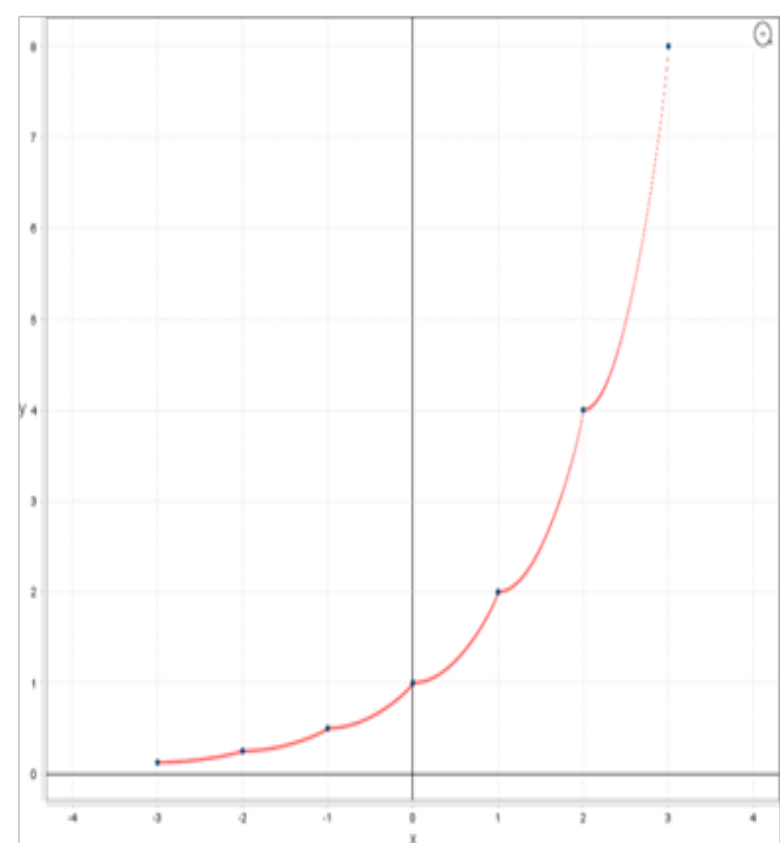

Figure 17 PFC 2D modeling for $\gamma=\sin ^{s}\left(\alpha \cdot \frac{\pi}{2}\right), s=0,8, h=0$.

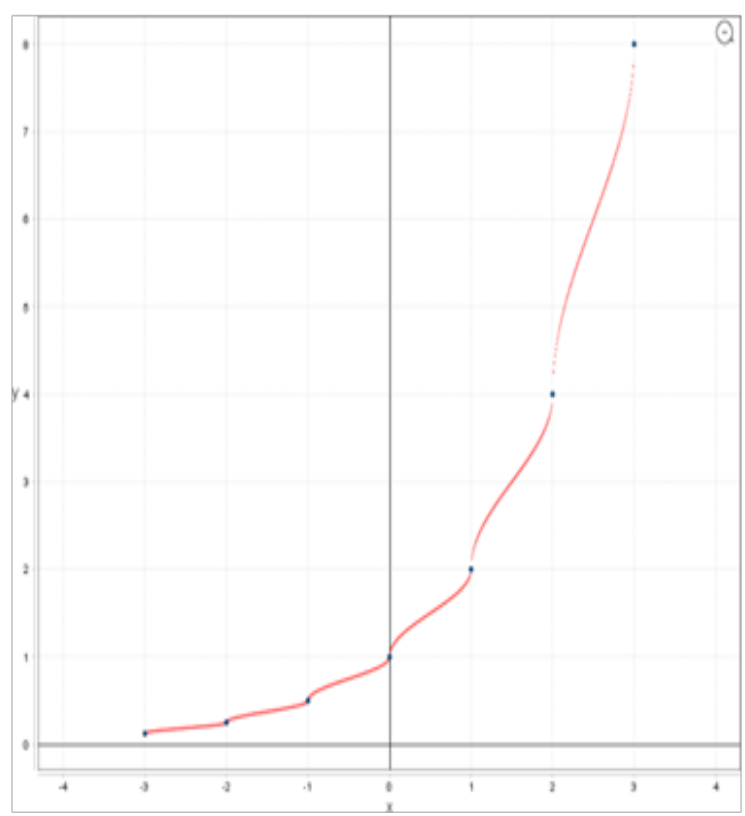

Figure 18 PFC 2D modeling for $\gamma=1-\frac{2}{\pi} \arccos \left(\alpha^{s}\right), s=0,5, h=0$. Modeling and interpolation of non-typical curves and irregular shapes

PFC two dimensional interpolations and modeling enables to solve classic problem in numerical methods for example (Figure 2) how to parameterize and to model known function. But having the set of nodes there is another problem connected with handwriting and 
human signing: how to model or to reconstruct the curve which is the part of signature or handwriting but which is non-typical or irregular. Human signature and handwriting consists of non-typical curves and irregular shapes. PFC method (1) is the way of modeling and interpolation for non-typical curves and irregular shapes - contours as closed curves (if first node and last node is the same). Here are some examples of modeled non-typical or irregular curves as a part of signature or handwriting for five nodes:

$(-0.9 ; 4.736),(-0.5 ; 0.666),(0 ; 0),(0.5 ;-0.666),(0.9 ;-4.736)$

Figures 22-24 are the examples of very specific modeling for nontypical and irregular curves as a signature. PFC interpolation is used for parameterization and reconstruction of curves in the plane.

\begin{tabular}{|l|l|l|l|l|l|l|l|l|}
\hline 2 & 3 & 0 & 0 & 0 & 0 & 0 & 0 & 0 \\
\hline 0 & 0 & 4 & 5 & 0 & 0 & 0 & 0 & 0 \\
\hline 0 & 0 & 0 & 0 & 6 & 0 & 0 & 0 & 0 \\
\hline 0 & 0 & 0 & 0 & 0 & 7 & 0 & 0 & 0 \\
\hline 0 & 0 & 0 & 0 & 0 & 0 & 8 & 0 & 0 \\
\hline 0 & 0 & 0 & 0 & 0 & 0 & 0 & 9 & 0 \\
\hline 0 & 0 & 0 & 0 & 0 & 0 & 0 & 0 & 10 \\
\hline 0 & 0 & 0 & 0 & 0 & 0 & 0 & 0 & 10 \\
\hline 0 & 0 & 0 & 0 & 0 & 0 & 0 & 0 & 10 \\
\hline
\end{tabular}

Figure 19 Reconstructed speed of the writing $s$ at the pixels of signature.

\begin{tabular}{|l|l|l|l|l|l|l|l|l|}
\hline 2,1 & 3,1 & 0 & 0 & 0 & 0 & 0 & 0 & 0 \\
\hline 0 & 0 & 4,2 & 5,2 & 0 & 0 & 0 & 0 & 0 \\
\hline 0 & 0 & 0 & 0 & 6,3 & 0 & 0 & 0 & 0 \\
\hline 0 & 0 & 0 & 0 & 0 & 7,4 & 0 & 0 & 0 \\
\hline 0 & 0 & 0 & 0 & 0 & 0 & 8,5 & 0 & 0 \\
\hline 0 & 0 & 0 & 0 & 0 & 0 & 0 & 9,6 & 0 \\
\hline 0 & 0 & 0 & 0 & 0 & 0 & 0 & 0 & 10,7 \\
\hline 0 & 0 & 0 & 0 & 0 & 0 & 0 & 0 & 10,8 \\
\hline 0 & 0 & 0 & 0 & 0 & 0 & 0 & 0 & 10,9 \\
\hline
\end{tabular}

Figure 20 Reconstructed pen pressure $p$ and speed of the writing $s$ as $(p, s)$ at the pixels of signature.

\begin{tabular}{|l|l|l|l|l|l|l|l|l|}
\hline $2,1,30$ & $3,1,30$ & 0 & 0 & 0 & 0 & 0 & 0 & 0 \\
\hline 0 & 0 & $4,2,32$ & $5,2,34$ & 0 & 0 & 0 & 0 & 0 \\
\hline 0 & 0 & 0 & 0 & $6,3,37$ & 0 & 0 & 0 & 0 \\
\hline 0 & 0 & 0 & 0 & 0 & $7,4,43$ & 0 & 0 & 0 \\
\hline 0 & 0 & 0 & 0 & 0 & 0 & $8,5,45$ & 0 & 0 \\
\hline 0 & 0 & 0 & 0 & 0 & 0 & 0 & $9,6,46$ & 0 \\
\hline 0 & 0 & 0 & 0 & 0 & 0 & 0 & 0 & $10,7,53$ \\
\hline 0 & 0 & 0 & 0 & 0 & 0 & 0 & 0 & $10,8,56$ \\
\hline 0 & 0 & 0 & 0 & 0 & 0 & 0 & 0 & $10,9,60$ \\
\hline
\end{tabular}

Figure 2 I Reconstructed pen pressure $p$, speed of the writing $s$ and angle a as $(\mathrm{p}, \mathrm{s}, \mathrm{a})$ at the pixels of signature.

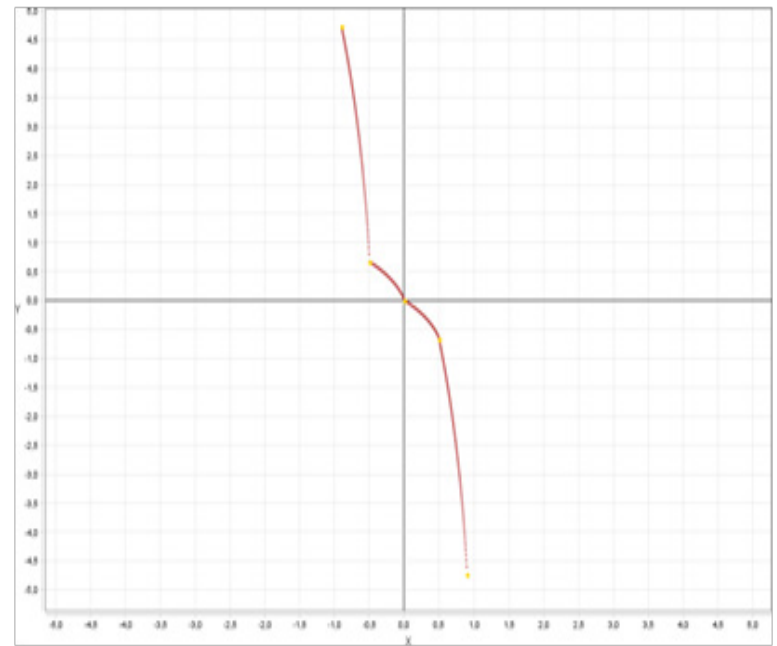

Figure 22 PFC 2D interpolation for $\gamma=\log _{2}{ }^{s}(\alpha+1), s=0.8$,

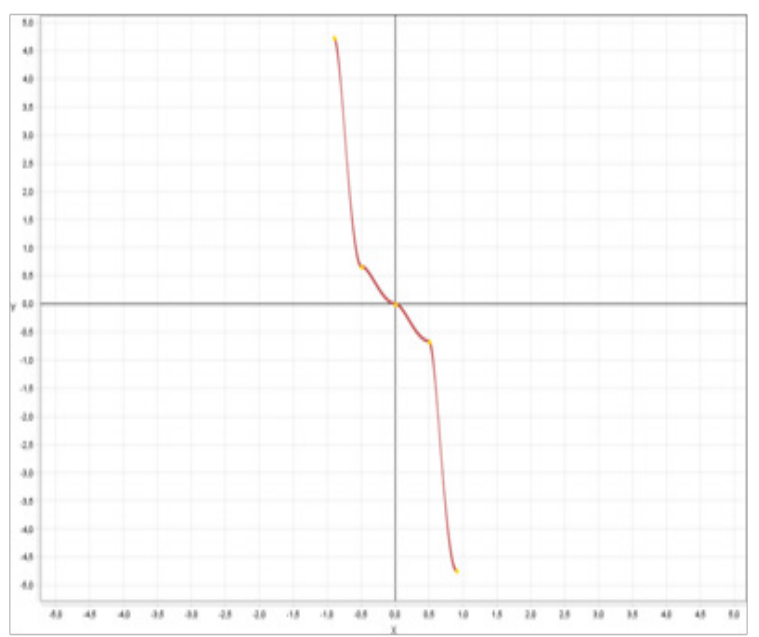

Figure $23 \mathrm{PFC} 2 \mathrm{D}$ interpolation for $\gamma=\sin \left(\alpha^{s} * \frac{\pi}{2}\right), s=1.8$,

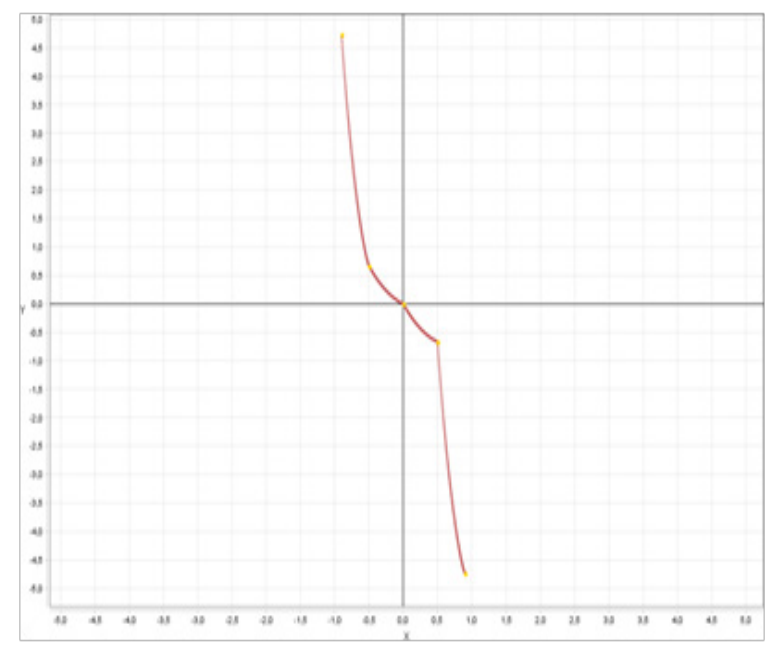

Figure 24 PFC 2D interpolation for $\gamma=\left(2^{\alpha}-1\right)^{s}, s=1.2, h=0$. 


\section{Conclusion}

The method of Probabilistic Features Combination (PFC) enables interpolation and modeling of high-dimensional $N$ data using features' combinations and different coefficients $\gamma$ : polynomial, sinusoidal, cosinusoidal, tangent, cotangent, logarithmic, exponential, arc sin, arc cos, arc tan, arc cot or power function. Functions for $\gamma$ calculations are chosen individually at each data modeling and it is treated as $N$-dimensional probability distribution function: $\gamma$ depends on initial requirements and features' specifications. PFC method leads to data interpolation as handwriting or signature identification and image retrieval via discrete set of feature vectors in $\mathrm{N}$ - dimensional feature space. So PFC method makes possible the combination of two important problems: interpolation and modeling in a matter of image retrieval or writer identification. Main features of PFC method are: PFC interpolation develops a linear interpolation in multidimensional feature spaces into other functions as $N$-dimensional probability distribution functions; PFC is a generalization of MHR method and PNC method via different nodes combinations; interpolation of $L$ points is connected with the computational cost of rank $O(L)$ as in MHR and PNC method; nodes combination and coefficients $\gamma$ are crucial in the process of data probabilistic parameterization and interpolation: they are computed individually for a single feature. Future works are going to applications of PFC method in signature and handwriting biometric recognition: choice and features of nodes combinations $h$ and coefficients $\gamma$.

\section{Acknowledgments}

None.

\section{Conflicts of interest}

Author declares that there is no conflict of interest.

\section{References}

1. Marti UV, Bunke H. The IAM-database: an English sentence database for offline handwriting recognition. Int $J$ Doc Anal Recognit. 2002;5(1):39-46.

2. Djeddi C, Souici-Meslati L. A texture based approach for Arabic writer identification and verification. International Conference on Machine and Web Intelligence. 2010. p. 115-120.

3. Djeddi C, Souici-Meslati L. Artificial immune recognition system for Arabic writer identification. International Symposium on Innovation in Information and Communication Technology. 2011. p. 159-165.

4. Nosary A, Heutte L, Paquet T. Unsupervised writer adaption applied to handwritten text recognition. Pattern Recogn Lett. 2004;37(2):385-388.

5. Van EM, Vuurpijl L, Franke K, et al. The WANDA measurement tool for forensic document examination. JForensic Doc Exam. 2005;16:103-118.

6. Schomaker L, Franke K, Bulacu M. Using codebooks of fragmented connected-component contours in forensic and historic writer identification. Pattern Recogn Lett. 2007;28(6):719-727.

7. Siddiqi I, Cloppet F, Vincent N. Contour based features for the classification of ancient manuscripts. Conference of the International Graphonomics Society. France; 2009. p. 226-229.

8. Garain U, Paquet T. Off-line multi-script writer identification using AR coefficients. International Conference on Document Analysis and Recognition. 2009. p. 991-995.
9. Bulacu M, Schomaker L, Brink A. Text-independent writer identification and verification on off-line Arabic handwriting. International Conference on Document Analysis and Recognition. 2007. p. 769-773.

10. Ozaki M, Adachi Y, Ishii N. Examination of effects of character size on accuracy of writer recognition by new local arc method. International Conference on Knowledge Based Intelligent Information and Engineering Systems. 2006. p. 1170-1175.

11. Chen J, Lopresti D, Kavallieratou E. The impact of ruling lines on writer identification. International Conference on Frontiers in Handwriting Recognition. USA; 2010. p. 439-444.

12. Chen J, Cheng W, Lopresti D. Using perturbed handwriting to support writer identification in the presence of severe data constraints. Document Recognition and Retrieval. 2011. p. 1-10.

13. Galloway MM. Texture analysis using gray level run lengths. Comput Graphics Image Process. 1975;4(2):172-179.

14. Siddiqi I, Vincent N. Text independent writer recognition using redundant writing patterns with contour-based orientation and curvature features. Pattern Recogn Lett. 2010;43(11):3853-3865.

15. Ghiasi G, Safabakhsh R. Offline text-independent writer identification using codebook and efficient code extraction methods. Image and Vision Computing. 2013;31(5):379-391.

16. Shahabinejad F, Rahmati M. A new method for writer identification and verification based on Farsi/Arabic handwritten texts. 9th International Conference on Document Analysis and Recognition (ICDAR 2007). 2007. p. 829-833.

17. Schlapbach A, Bunke H. A writer identification and verification system using HMM based recognizers. Pattern Anal Appl. 2007;10(1):33-43.

18. Schlapbach A, Bunke H. Using HMM based recognizers for writer identification and verification 9th Int. Workshop on Frontiers in Handwriting Recognition. 2004. p. 167-172.

19. Schlapbach A, Bunke H. Off-line writer identification using Gaussian mixture models. International Conference on Pattern Recognition. China; 2006. p. 992-995.

20. Bulacu M, Schomaker L. Text-independent writer identification and verification using textural and allographic features. IEEE Trans Pattern Anal. Mach Intell. 2007;29(4):701-717.

21. Schumaker LL. Spline Functions: Basic Theory. 3rd ed. Cambridge Mathematical Library, UK; 2007

22. Collins I. Fundamental Numerical Methods and Data Analysis. Case Western, Reserve University, USA; 2003. p. 1-283.

23. Chapra SC. Applied Numerical Methods. India: McGraw-Hill; 2012.

24. Ralston A, Rabinowitz P. A First Course in Numerical Analysis. 2nd ed. New York: Dover Publications; 2001.

25. Zhang D, Lu G. Review of Shape Representation and Description Techniques. Pattern Recognition. 2004;1(37):1-19.

26. Jakóbczak DJ. 2D Curve Modeling via the Method of Probabilistic Nodes Combination - Shape Representation, Object Modeling and Curve Interpolation-Extrapolation with the Applications 1st ed. Germany: LAP Lambert Academic Publishing; 2014. 